\title{
Commentary: “Fungal Infections of Oral Cavity: Diagnosis, Management, and Association with COVID-19"
}

\author{
Ambadasu Bharatha ${ }^{1} \cdot$ Latha Kandamaran $^{2} \cdot$ Kandamaran Krishnamurthy $^{3}$ (D)
}

Accepted: 3 July 2021 / Published online: 10 July 2021

(C) The Author(s), under exclusive licence to Springer Nature Switzerland AG 2021

The recently published article by Rajendra Santosh et al. [2021] elegantly addressed on diagnosis and management of oral fungal infections. The authors also mentioned the association of oral fungal infections in COVID-19 patients. Oral candidiasis is the only condition mentioned in such association with COVID-19 infection [1]. However, there is a sudden surge in pulmonary, and rhinocerebral (paranasal sinus, oral cavity, and brain) types of mucormycosis are observed in COVID-19 infections [2]. Thus, mucormycosis should also be added to the list of associated oral fungal conditions in COVID 19 infections.

Coronavirus disease 2019 (COVID-19) continues to be a major health concern across the world. COVID-19 infection causes mild to life-threatening infections with an association of pneumonia and bacterial and fungal coinfections. Individuals with diabetes, chronic obstructive pulmonary disease (COPD), or patients with ventilator in intensive care unit (ICU), immunocompromised due to steroid therapy are likely to develop severe opportunistic infections. COVID-19 patients who receive steroids, monoclonal antibodies, or broad-spectrum antibiotic therapy are

This article is part of the Topical Collection on Covid-19

Kandamaran Krishnamurthy

getdrkandy@yahoo.com

Ambadasu Bharatha

ambadasu.bharatha@ cavehill.uwi.edu

Latha Kandamaran

getdrlatha@gmail.com

1 Lecturer in Pharmacology, Faculty of Medical Sciences, University of the West Indies, Cave Hill, Barbados

2 Associate Lecturer in Radiology, Faculty of Medical Sciences, University of the West Indies, Cave Hill, Barbados

3 Senior Associate Lecturer in Pediatrics, Faculty of Medical Sciences, University of the West Indies, Cave Hill, Barbados inclined to another layer of risk for developing a fungal infection or exacerbate pre-existing conditions [3].

The pathogenesis of fungal infection is linked to alteration of T-cell number in COVID-19 infection. In addition, specific pathophysiologic characteristics of the COVID-19 condition may also allow secondary fungal infections. This specific pathophysiologic characteristic change occurs due to severe lung illness with alveolo-interstitial pathology. Thus, COVID19-mediated immunological dysregulation leads to the downregulation of $\mathrm{T}$ lymphocytes (both $\mathrm{CD} 4+\mathrm{T}$ and $\mathrm{CD} 8+\mathrm{T}$ cells) and alters innate immunity [4].

Sinuses, lungs, and skin are the most reported sites of invasive mucormycosis [5]. Signs and symptoms depend on the site of the infection. In oral mucormycosis, throbbing pain related to tongue and oropharynx with multiple mediumsized pseudomembranous structures with white plaques scattered over the dorsal surface of the tongue causing glossitis; and over the hard palate, mouth floor, soft palate, oropharynx region, and to a lesser extent the buccal mucosa which resembles oropharyngeal candidiasis (OPC) [6].

Mucormycosis may cause necrotic cutaneous lesions in immunocompromised patients. However, other infections such as Aspergillus, Fusarium, Pseudallescheria, and Scedosporium species should be considered in the differential diagnosis. Ecthyma gangrenosum caused by Pseudomonas aeruginosa can also present a similar appearance. Despite the non-specific nature of the clinical signs and symptoms, some of them may have a high predictive value. A history of voriconazole prophylaxis or the onset of breakthrough fungal infection while receiving antifungal medications effective against Aspergillus but not to Mucorales indicates the presence of mucormycosis in a susceptible host [7].

Amphotericin B is the most frequently used medication for the management of mucormycosis [1]. Kidney function tests should be investigated on individuals with amphotericin $\mathrm{B}$ due to the association of increased risk of nephrotoxicity. Combination therapy includes echinocandins and amphotericin B as a second line of management in severe 
mucormycosis cases. The combination of echinocandins with amphotericin enhances therapeutic success due to poly backbone formation. In addition to the above two drugs in medication management, triazoles, posaconazole, or isavuconazole are also considered as the next line of drug in the management of mucormycosis [8]. Antifungal action of triazoles is achieved by inhibition of 14-demethylation, which causes an elevation of toxic 14-methyl sterols levels, which alters the permeability of the fungal membrane. Posaconazole is preferred when amphotericin B intolerance is observed. Hence, posaconazole remains as a single choice of antifungal medication for treating invasive mucormycosis when amphotericin B intolerance is observed [9]. The optimal treatment duration for mucormycosis still remains unknown, and the duration of treatment may be decided by the clinical response and tolerance of the treatment regimen.

The development of mucormycosis is most likely due to the use of steroids, which cautions its use in COVID-19 patients. Even though steroids are theoretically beneficial in delaying progression in some patients, it seems illogical to take them in all patients with persistent and recurrent mild COVID-19 fever to "prevent" hypoxia-when hypoxia is only found in a minority of patients, and no research has proven that prophylactic steroids are effective in preventing the progression of mild COVID-19 patients [10]. Therefore, the use of steroids in mild to moderate COVID-19 patients (without hypoxemia) or administration of higher steroid doses should be avoided. It is worthwhile to state that early diagnosis of mucormycosis and initiation of appropriate antifungal medication are necessary to minimize morbidity (end-organ damage) and reduce mortality. Medications that target immune pathways, such as tocilizumab, should also be avoided unless a significant benefit is proven [11]. Careful monitoring is necessary on critically ill COVID-19 patients due to the association of serious infections that worsen the existing disease. The diagnosis of pulmonary mucormycosis necessitates a high level of clinical suspicion. For achieving better prognostic outcomes in COVID-19 individuals with pulmonary mucormycosis, early diagnosis and treatment are essential.

\section{Availability of Data and Material Not applicable}

Code Availability (Software Application or Custom Code) Not applicable

Author Contribution All the three authors contributed in this writeup.

\section{Declarations}

Ethics Approval Not applicable

Consent to Participate Not applicable

Consent for Publication Not applicable

Conflict of Interest The authors declare no competing interests.

\section{References}

1. Rajendra Santosh AB, Muddana K, Bakki SR. Fungal infections of oral cavity: diagnosis, management, and association with COVID19 [published online ahead of print]. SN Compr Clin Med. 2021:112.

2. Nehara HR, Puri I, Singhal V, Ih S, Bishnoi BR, Sirohi P. Rhinocerebral mucormycosis in COVID-19 patient with diabetes a deadly trio: case series from the north-western part of India. Indian J Med Microbiol. 2021;S0255-0857(21):04111-6.

3. Mehta S, Pandey A. Rhino-orbital mucormycosis associated with COVID-19. Cureus. 2020;12(9):e10726.

4. Gangneux JP, Bougnoux ME, Dannaoui E, Cornet M, Zahar JR. Invasive fungal diseases during COVID-19: we should be prepared. J Mycol Med. 2020;30(2):100971.

5. Torres-Narbona M, Guinea J, Martínez-Alarcón J, Muñoz P, Gadea I. Bouza E; MYCOMED Zygomycosis Study Group. Impact of zygomycosis on microbiology workload: a survey study in Spain. J Clin Microbiol. 2007;45(6):2051-3.

6. Riad A, Gomaa E, Hockova B, Klugar M. Oral candidiasis of COVID-19 patients: case report and review of evidence. J Cosmet Dermatol. 2021;20:1580-4.

7. Skiada A, Pavleas I, Drogari-Apiranthitou M. Epidemiology and diagnosis of mucormycosis: an update. J Fungi (Basel). 2020;6(4): 265.

8. Spellberg B, Ibrahim AS, Chin-Hong PV, Kontoyiannis DP, Morris MI, Perfect JR, et al. The deferasirox-ambisome therapy for mucormycosis (DEFEAT Mucor) study: a randomized, doubleblinded, placebo-controlled trial. J Antimicrob Chemother. 2012;67(3):715-22.

9. Riley TT, Muzny CA, Swiatlo E, Legendre DP. Breaking the mold: a review of mucormycosis and current pharmacological treatment options. Ann Pharmacother. 2016;50(9):747-57.

10. Ray A, Goel A, Wig N. Corticosteroids for treating mild COVID19: opening the floodgates of therapeutic misadventure. QJM: An International Journal of Medicine. 2021:hcab138.

11. Kimmig LM, Wu D, Gold M, Pettit NN, Pitrak D, Mueller J, et al. IL-6 inhibition in critically Ill COVID-19 patients is associated with increased secondary infections. Front Med (Lausanne). 2020;7: 583897.

Publisher's Note Springer Nature remains neutral with regard to jurisdictional claims in published maps and institutional affiliations. 